\title{
Inovação na produção científica brasileira em gestão e negócios: mapeamento bibliométrico nas bases Web of Science e Scopus
}

A inovação é amplamente difundida como um mecanismo capaz de promover o desenvolvimento da sociedade através da criação de novos aparatos tecnológicos e melhoria de técnicas utilizadas na atualidade. No Brasil, o tema ganhou marco legal com a publicação da Lei 10.973, de 2 de dezembro de 2004 (Lei da Inovação), e a partir daí mecanismos para uma série de investimentos públicos e privados. Nessa perspectiva, o presente estudo objetivou avaliar qual o impacto na produção cientifica brasileira sobre inovação e negócios acerca da inovação após a implementação da referida lei. Utilizou-se, portanto, um estudo bibliométrico com a string de busca "TS = (Innovation*) AND CU = BRA? I.*” em um espaço temporal de 2005 até 2019 nas bases de dados da Web of Science e Scopus. O estudo evidenciou um crescimento das pesquisas sobre a temática, partindo números entre 2 e 7 no ano de 2005 para números como 230 e 288 em 2018, na Scopus e Web of Science, respectivamente. Entre às áreas de publicação, se evidenciou que as áreas de gestão e negócios concentram a maior parcela de publicações, sendo 288 em 2018, na Scopus e Web of Science, respectivamente. Entre às áreas de publicação, se evidenciou que as áreas de gestão e negócios concentram a maior parcela de publicaç̃̃es, sendo a maioria publicado em formato de artigo nas duas plataformas. A instituição que mais se destacou no estudo foi a Universidade de São Paulo (USP), sendo a que mais publicou em ambos as bases de dados. Observou-se também que as revistas que mais publicam sobre o tema são a Espacios e a Geintec, sendo a professora do Centro Universitário UNIFBV/Wyden Eliana Andrea Severo e o professor da USP Felipe Mendes Borini os pesquisadores brasileiros que mais se destacam na área, com 21 e 15 publicações, na Scopus e Web of Science, respectivamente. Porém, estudo bri o sobre a temática após a promulgação da Lei da Inovação, e que possui instituições que produzem bastante sobre a temática, mas ainda evidenciou a necessidade dos pesquisadores brasileiros de recorrem a fontes internacionais para embasar seus estudos.

\section{Innovation in Brazilian scientific production in management and business: bibliometric mapping in the Web of Science and Scopus databases}

\begin{abstract}
Innovation is widely disseminated as a mechanism capable of promoting the development of society through the creation of new technological devices and improvement of techniques used today. In Brazil, the topic gained legal status with the publication of Law 10,973, of December 2, 2004 (Law of Innovation), and from there mechanisms for a series of public and private investments. In this perspective, the present study aimed to assess what is the impact on Brazilian scientific production on innovation and business regarding innovation after the implementation of that law. Therefore, a bibliometric study with the search string "TS = (Innovation *) AND CU = BRA? II" * was used in a period from 2005 to 2019 in the Web of Science and implementation of that law. Therefore, a bibliometric study with the search string "TS = (Innovation *) AND CU = BRA? II" was used in a period from 2005 to 2019 in the Web of Science and
Scopus databases. The study showed an increase in research on the theme, breaking numbers between 2 and 7 in 2005 to numbers like 230 and 288 in 2018 , in Scopus and Web of Science, respectively. Among the areas of publication, it was evident that the areas of management and business concentrate the largest share of publications, with the majority being published in article format on both platforms. The institution that stood out the most in the study was the University of São Paulo (USP), being the one that most published in both databases. It was also observed that the magazines that publish the most on the topic are Espacios and Geintec, with the professor at the UNIFBV/Wyden University Center Eliana Andrea Severo and the professor at USP Felipe Mendes Borini being the Brazilian researchers who most stand out in the area, with 21 and 15 publications, in Scopus and Web of Science, respectively. However, among the studies most cited by Brazilian researchers, there was still a predominance of the use of international references, and the publications most cited by Brazilian studies in both databases are of international authorship, even with Brazilian collaboration in specific cases. The study, therefore, evidenced the growing interest on the theme after the enactment of the Innovation Law, and which has institutions that produce a lot on the theme, but it also evidenced the need for Brazilian researchers to use international sources to support their studies.
\end{abstract}

Keywords: Bibliometry; Innovation Studies; Brazilian researchers.

\section{Topic: Inovação Tecnológica}

Reviewed anonymously in the process of blind peer.
Received: $17 / 04 / 2021$

Approved: 10/06/2021

Edivaldo Rabelo Menezes (iD

Universidade Federal de Sergipe, Brasil

http://lattes.cnpq.br/2541866097413422

http://orcid.org/0000-0002-7144-3795

edivaldorabelo@uern.br

Antônia Rafaela Pamola Mota Fernandes (iD

Universidade do Estado do Rio Grande do Norte, Brasil

http://lattes.cnpq.br/8758700456806909

http://orcid.org/0000-0001-6857-4367

arafaelapmf@gmail.com

Francisco Souza Rego Filho (iD)

Universidade do Estado do Rio Grande do Norte, Brasil

http://lattes.cnpq.br/4209058471389671

http://orcid.org/0000-0002-8701-2156

filhosouzafs@gmail.com

Referencing this:

MENEZES, E. R.; FERNANDES, A. R. P. M.; REGO FILHO, F. S.. Inovação na produção científica brasileira em gestão e negócios: mapeamento bibliométrico nas bases Web of Science e Scopus. Revista Brasileira de Administração Científica, v.12, n.2, p.300-311, 2021. DOI: http://doi.org/10.6008/CBPC2179-684X.2021.002.0024 


\section{INTRODUÇÃO}

Inovação é um termo recorrentemente utilizado no mundo dos negócios e na área administrativa como um todo, com a globalização e o avanço tecnológico a inovação se tornou uma peça chave para o desenvolvimento e crescimento das organizações, visto que fatores como concorrência, crises econômicas, abertura de novos mercados, fizeram com que as empresas tivessem que se reinventar para permanecerem competitivas e sustentáveis (FUCK et al., 2012).

O Manual de Oslo, uma das principais referências de estudo sobre inovação, a define como sendo a implementação de um produto, serviço, processo ou método novo, ou ainda significativamente melhorado (OCDE, 1997). Corroborando com Schumpeter (1939) que conceitua o processo inovativo como sendo a realização de novas combinações que irão gerar novas versões de um produto/serviço já existentes (COSTA et al., 2017).

No Brasil, os primeiros passos ao desenvolvimento da Ciência, Tecnologia e Inovação (CT\&I) começa ainda na década de 50, com a criação de órgãos reguladores como o Conselho Nacional de Desenvolvimento Científico e Tecnológico (CNPq) responsáveis por criar políticas públicas de incentivo e execução de projetos para consolidação de um sistema de CT\&I (BUFREM et al., 2018).

Mas foi a partir de 2004 que uma nova conjuntura política se estabeleceu no que diz respeito ao incentivo das atividades de Pesquisa e Desenvolvimento (P\&D), sendo considerado o marco legal da inovação no Brasil, com a criação da Lei 10.973, de 2 de dezembro de 2004 (Lei da Inovação) e da Lei 11.196, de 21 de novembro de 2005 (Lei do Bem) (CNI, 2018).

Porém, o baixo desempenho do país em relação a competividade global em termos de inovação está atrelado à ausência de dispositivos legais mais eficientes e práticos, onde estes deveriam ser responsáveis por subsidiar o acesso das empresas a inovação através de uma melhor implementação de incentivos fiscais, qualificação de mão de obra especializada, e disponibilização de recursos para gerar poder de compra e montagem de infraestrutura que permitam a transferência de tecnologia das universidades e instituições de pesquisa para as empresas, e consequentemente, para o mercado ao desenvolver a capacidade de inovar na criação de novos produtos/serviços e implementação de novos processos.

Diante do exposto, o objetivo do presente artigo é analisar o avanço da pesquisa cientifica sobre inovação em gestão e negócios, através de uma bibliometria realizadas nas bases Web of Science e Scopus, com marco temporal de 2005 a 2019, para melhor entender o contexto da inovação na pesquisa cientifica brasileira na área de negócios, especificamente após publicação da Lei no 10.973/2004 (Lei da Inovação).

\section{REVISÃO TEÓRICA}

\section{Inovação}

A ideia de inovação sempre está ligada a algo novo ou significativamente melhorado, porém seu conceito é multifacetado, pois cada área do conhecimento que adota o processo de inovação tem um objetivo distinto a ser atingido. Na área de marketing por exemplo a inovação está voltada para a capacidade 
criativa da comunicação, com intuito de atingir um público alvo, já no meio empresarial é vista como um processo de criação de novas ideias com potencial para se transformarem em produtos, serviços que solucionem problemas escaláveis gerando negócios rentáveis (CARDOSO, 2010).

Schumpeter (1939) um dos primeiros estudiosos da área, apresenta inovação como um elemento propulsor da economia, tendo em vista os avanços causados pela globalização como: abertura de novos mercados, criação de novas organizações comerciais, surgimento de novos produtos, e a conceituou como sendo o processo de fazer coisas de maneira diferente através de combinações de recursos já existentes, considerando a inovação um mecanismo fundamental para a sobrevivência dos negócios a nova realidade do mercado.

Complementar ao conceito Schumpter (1939), o Manual de Oslo (OCDE, 1997), considerada a principal fonte internacional sobre atividades inovadora da indústria, diz que inovação nada mais é do que a implementação de um produto, serviço, processo ou método novo em uma organização.

No entanto, um conceito que aparentemente parece simples, por se tratar de algo novo, o processo de construção da inovação é um tanto complexo e exige a participação não só da empresa que são centrais nesse processo, mas também dos detentores de conhecimento que são os pesquisadores (AMARAL, 2015). O Manual de Oslo classifica a inovação em 4 tipos, tal como exposto no Quadro 1.

Quadro 1: Tipos de Inovação.

\begin{tabular}{|l|l|}
\hline INOVAÇÕES DE: & CONCEITUAÇÃO \\
\hline PRODUTO & Quando há mudanças significativas na formatação do produto, envolvendo a sua formula. \\
\hline PROCESSO & Referem-se as mudanças que ocorrem na execução e técnica de produção; \\
\hline MARKETING & Mudanças no design, na apresentação e comunicação do produto com seu público alvo. \\
\hline ORGANIZACIONAIS & $\begin{array}{l}\text { Diz respeito a mudanças no layout da empresa, nas técnicas de trabalho, inclusão de modernos } \\
\text { equipamentos. }\end{array}$ \\
\hline
\end{tabular}

Fonte: OCDE (1997).

Uma empresa busca inovar por diversos motivos, dentre eles se pode citar exemplos tais como gerar maior atratividade para o seu produto, se destacar da concorrência e atingir maiores fatias de mercado, todas essas estratégias buscando um foco determinado, o alcance de uma maior geração de receita.

A partir disso, adota-se uma cultura de inovação dentro e fora das empresas, visto o impacto dos resultados da inovação no mundo empresarial surgem os habitats de inovação para dá suporte ao processo inovativo, surgindo como instrumentos de políticas públicas responsáveis pelo incentivo e desenvolvimento da inovação a nível local, regional e mundial tendo como principal objetivo promover a integração entres institutos de pesquisa, intuições de ensino superior (IES), governo e a iniciativa privada (DEPINE et al., 2018).

Visto que o conhecimento se tornou a principal fonte de inovação para as empresas, diferente dos ativos físicos como maquinas e equipamentos, ele não é facilmente absorvida pelas empresas, pois requer aprendizado, esforço tecnológico, profissionais capacitados influenciando diretamente no processo de inovação das empresas, a transformação de conhecimento em inovação requer que as empresas tenham acesso a informações que dão origem a inovação, informações que provem sobre tudo de fornecedores, concorrentes, clientes, redes de informação.

O que inicialmente surgiu como uma necessidade das empresas de se reinventarem para 
permanecerem no mercado, com o surgimento e expansão dos ecossistemas de inovação que são ambientes propícios ao desenvolvimento e transferência de inovação das empresas para o mercado e para a sociedade (SEBRAE, 2020). Estes que se tornaram importantes mecanismos impulsionadores da inovação, abrindo espaço principalmente para pequenas e médias empresas se tornarem mais competitivas ao desenvolverem sua capacidade inovativa (WELTER et al., 2019).

\section{O marco legal da inovação no Brasil}

Uma pesquisa realizada pelo SEBRAE no ano de 2020, intitulada "Ecossistemas de Empreendedorismo Inovadores e Inspiradores" ao comparar a atuação de ecossistemas de inovação entre o Brasil e o mundo, identificou que o Brasil embora tenha uma maior intenção empreendedora, o acesso ao capital de risco dificulta a geração de novos negócios, enquanto os outros países estão mais preparados nesse aspecto (SEBRAE, 2020). É indiscutível que o Brasil não esteja mobilizado para inovação, inúmeras são as iniciativas no país, porém os esforços ainda são insuficientes quando comparados com o desenvolvimento de outros países, como bem mostrou o estudo citado, não há falta iniciativa e sim financiamentos para execução dos projetos (CARDOSO, 2010).

O marco da inovação no Brasil, acontece após cerca de duas décadas depois de pouco incentivo e estimulo ao desenvolvimento cientifico e tecnológico que foi o advento de alguns mecanismos legais, responsáveis por criar ações mais consistentes por parte do governo, instituições de CT\&I, empresas, como pode ser visto no Quadro 2.

Quadro 2: Recorte histórico das políticas de incentivo à inovação no Brasil.

\begin{tabular}{|l|l|}
\hline ANO & POLÍTICAS PÚBLICAS \\
\hline $\mathbf{1 9 5 0}$ & Coordenação de Aperfeiçoamento de Pessoal de Nível Superior (CAPES) \\
\hline $\mathbf{1 9 5 1}$ & Conselho Nacional de Desenvolvimento Científico e Tecnológico (CNPq) \\
\hline $\mathbf{1 9 5 2}$ & Criação do Banco Nacional de Desenvolvimento Social (BNDES) \\
\hline $\mathbf{1 9 6 7}$ & Criação da Financiadora de Estudos e Projetos (FINEP) \\
\hline $\mathbf{1 9 7 3}$ & Empresa Brasileira de Pesquisa Agropecuária (Embrapa) \\
\hline $\mathbf{1 9 8 5}$ & Ministério da Ciência e Tecnologia (MCT) \\
\hline $\mathbf{1 9 9 6}$ & Lei de propriedade industrial \\
\hline $\mathbf{2 0 0 1}$ & Lei dos fundos setoriais CT\&I \\
\hline $\mathbf{2 0 0 3}$ & Lei de Biossegurança \\
\hline $\mathbf{2 0 0 3}$ & Lei da Política Industrial Tecnológica Exterior (PITCE) \\
\hline $\mathbf{2 0 0 4}$ & Lei da Inovação \\
\hline $\mathbf{2 0 0 5}$ & Lei da Política de Desenvolvimento produtivo \\
\hline $\mathbf{2 0 0 5}$ & Lei do Bem \\
\hline $\mathbf{2 0 0 7}$ & Programa de Apoio à Capacitação Tecnológica da Indústria (PACTI) \\
\hline $\mathbf{2 0 0 8}$ & Plano de Desenvolvimento Produtivo (PDP) \\
\hline $\mathbf{2 0 1 1}$ & Plano Brasil Maior (PBM) \\
\hline $\mathbf{2 0 1 2}$ & Estratégia nacional de ciência, tecnologia e inovação (ENCTI) \\
\hline
\end{tabular}

Fonte: Elaborado com base em Cardoso (2010).

Está claro que a inovação para acontecer dentro das empresas, principalmente as que requerem alta tecnologia dependem de políticas de incentivo, capital humano e práticas gerenciais, apesar de várias políticas de incentivo, o Brasil tem baixa produção de patentes e gastos em $P \& D$, principalmente no setor privado, o que mostra a dependência que o desenvolvimento da inovação nas pequenas e médias empresas no Brasil tem do setor público, através do fomento à inovação. Tendo em vista que o governo pode atuar de 
três formas no fomento ao processo inovativo: através do investimento em infraestrutura básica de CT\&l, apoio indireto com incentivos fiscais e na criação de políticas de apoio direto hoje no Brasil os fundos de financiamento inovativo representam um dos mais importantes instrumentos de política de inovação.

De acordo com a Pesquisa de Inovação PINTEC, que é uma pesquisa que acontece a cada 3 anos fazendo um levantamento sobre as atividades de inovação no Brasil, o último estudo realizado no ano de 2017, mostrou que 39.329 empresas implementaram inovações de produto ou processo no Brasil, e foram investidos cerca de R\$ 67, 3 bilhões de reais em inovação. Porém a taxa de inovação no país vem caindo, essas mesmas pesquisas realizadas em anos anteriores mostra o declínio de 2009-2011 (35,7\%) e 2012-2014 (36,0\%), o triênio $2015-2017$ registrou taxa de $33,6 \%$, um recuo de 2,4 pontos percentuais.

O impacto da Lei da inovação (Lei 10.973/2004) no país mostrou que a criação de dispositivos legais eficientes é fundamental para o desenvolvimento do sistema de inovação no Brasil, pois somente através de incentivos fiscais, acesso a infraestrutura e mão de obra qualificada, criação de uma rede de inovação que só é possível a partir do estimulo a cooperação entre universidades, empresas e instituições de pesquisa ampliando a capacidade das empresas de gerar inovações e alavancar a competitividade nacional.

Ainda segundo o mesmo estudo os obstáculos que mais impedem as empresas de inovar no Brasil, são: riscos econômicos excessivos, elevados custos de inovação, falta de pessoal qualificado, escassez de fontes de financiamento. Embora o Brasil ocupe o $9^{\circ}$ lugar no ranking de países que mais investem em inovação, isso no que diz respeito ao poder de compra, pois no ranking que leva em consideração o impacto dos investimentos em inovação no Produto Interno Bruto (PIB) o Brasil não aparece, o conjunto de obstáculos que impedem o Brasil de se tornar mais competitivo é a baixa capacidade de transformar a capacidade inovadora em produtos e serviços de forma que gere renda e influencie no PIB. Já no ranking internacional de inovação o Brasil ocupa a $66^{\circ}$ posição caindo duas posições, consequência da queda no investimento em Pesquisa e Desenvolvimento (P\&D).

\section{METODOLOGIA}

O presente estudo, trata-se de uma Bibliometria, técnica que pode ser definida como sendo uma análise da literatura, a partir da aplicação de técnicas estatísticas e matemáticas que resultará em um panorama da temática investigada de acordo com os indicadores previamente definido (CHUEKE et al., 2015; MOMESSO et al., 2017).

Para a realização desta pesquisa foram escolhidas duas bases de dados, a Web of Science, plataforma que contempla mais de 20.000 periódicos acadêmicas, e a Scopus, considerada o maior banco de dados de resumos e citações da literatura, por apresentar um panorama abrangente da produção cientifica mundial nas áreas de ciência, tecnologia, medicina, ciências sociais, artes e humanidades.

Para extração dos dados bibliométricos nas bases escolhidas, foram definidas as seguintes métricas apresentadas no Quadro 3. 
Quadro 3: Delineamento das métricas de pesquisa.

\begin{tabular}{|l|l|l|}
\hline BASES & Scopus & Web of Science \\
\hline PALAVRAS-CHAVE & "Innovation*” & TS = (Innovation*) AND CU = BRA?II* \\
\hline INTERVALO DE TEMPO & 2005 a 2019 & 2005 a 2019 \\
\hline ÁREA DE PESQUISA & Negócios, gestão e Contabilidade & Negócios, Administração pública, Finanças \\
\hline
\end{tabular}

Realizar um estudo bibliometrico a respeito da temática inovação nos permitiu entender como está sendo a evolução da produção cientifica nessa área, e mais especificamente neste estudo, após a criação da Lei da inovação $n^{\circ} 10.793$ em 2005, considerado o marco da inovação no Brasil, pois seu principal objetivo era alavancar os investimentos em inovação e pesquisa científica e tecnológica dentro das empresas, setor na qual se discutia sua fragilidade em políticas tecnológicas. Na coleta de dados identificou-se quais os autores que mais publicam com essa temática, quais instituições estão vinculadas, como está o avanço ao longo desses 14 anos, em quais periódicos estão sendo publicados.

\section{RESULTADOS E DISCUSSÃO}

A fim de compreender o avanço na área da produção científica sobre a temática Inovação no Brasil a partir do impacto da Lei da Inovação, de acordo com os dados coletados nas bases Web of Science e Scopus, como se pode observar Figura 1, houve um crescimento exponencial em ambas as bases de forma gradativa, sendo importante salientar que nas duas bases o pico de publicação foi entre os anos de 2014 e 2015, um dos fatores que influenciaram para isso, foi o aumento da produção cientifica brasileira, intitulado de 'boom cientifico' que ocorreram com o aumento dos investimentos em Ciência e tecnologia (C\&T).



Figura 1: Avanço das publicações científicas brasileira sobre Inovação (2005 a 2019)

Nessa perspectiva, embasam a maior necessidade de ampliar o número de publicações e a visibilidade das publicações na área pelo fato de o Brasil ocupar atualmente a $15^{\circ}$ posição entre os países que mais publicam artigos, porém no que diz respeito a citações o país ocupa a $34^{\circ}$ posição o que importante tanto quanto o número de publicações, pois é através do número de citações que se mede a relevância e a qualidade do estudo publicado.

O presente estudo limitou sua busca, focando-se na área de gestão e negócios, sendo assim, na base da Web of Science foram encontrados 1.239 publicações nessa área de concentração, enquanto na base Scopus foi um número um pouco maior com 1.803 resultados, ou seja, uma área de pesquisa que vem se mostrando em uma crescente, principalmente após a publicação da Lei da inovação em 2004, está que possibilitou maiores investimentos no setor e consequentemente um maior desencadeamento de interesse 
pelo estudo da área que começou a se expandir, como se pode verificar na Figura 2.

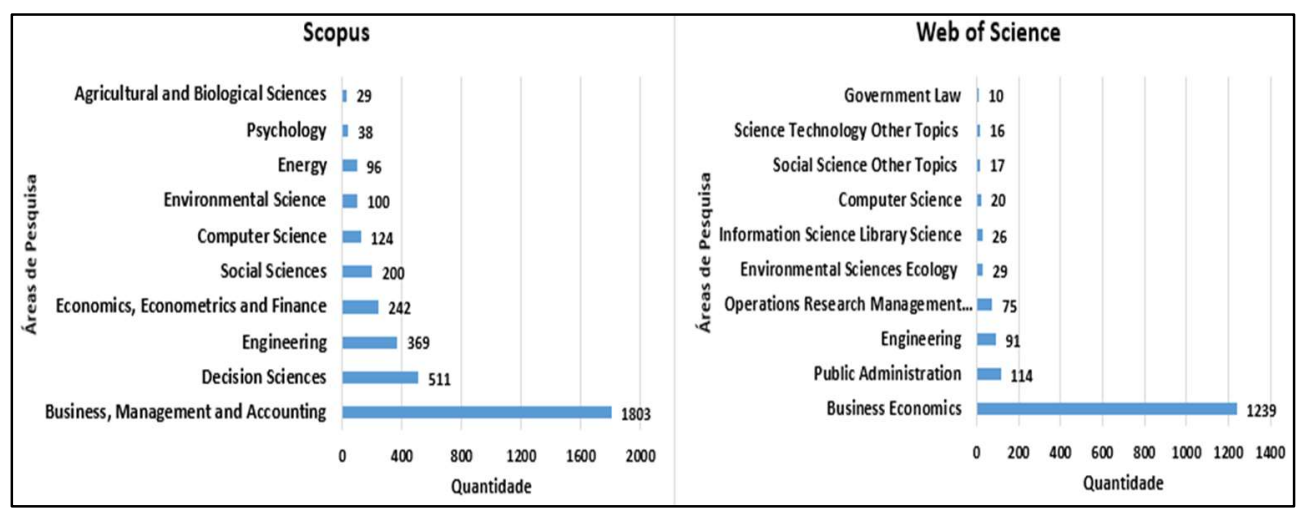

Figura 2 - Áreas de atuação da publicação científica brasileira sobre Inovação (2005 a 2019).

Tal predominância de publicações na área, em relação às demais listadas em ambas as bases de dados reforça a ideia de que inovação é um tema bastante alinhado com estudos organizacionais e voltados para o mercado competitivo, fazendo assim que a maioria dos seus estudos estejam vinculados diretamente às áreas como administração, negócios, economia e engenharia.

Dentre as diversas formas de documentos publicados, os artigos científicos se destacam como sendo a maior forma de publicação, estes que são mais completos em sua estrutura, pois apresentam e discutem ideias, métodos e resultados, como pode ser visto na Figura 3, sendo 1325 artigos na base Scopus e 1073 na Web of Science, tal como evidenciado na Figura 3.



Figura 3: Tipos de documentos sobre a publicação científica brasileira sobre Inovação (2005 a 2019). Fonte: Elaborado a partir de dados coletados da Web of Science e Scopus (2020).

Com relação aos periódicos que mais publicaram sobre o tema estudado, o periódico com o maior número publicações na Scopus é a revista Espacios com 316 publicações, um número significativamente maior do que o periódico que publicou na Web of Science, a Revista GEINTEC: Gestão, Inovação e Tecnologias, que conta com um total de 90 publicações no período, assim como observado na Figura 4.

O que chama atenção nesses dados é que os periódicos não são comuns entre as bases, aparecem exclusivamente em cada uma. A Espacios com uma característica multidisciplinar, um periódico latinoamericano, mas com 32\% das suas publicações vindas do Brasil e a GEINTEC concentrada nas áreas de gestão, inovação e tecnologia. Diferente da Web of Science na qual as publicações são em sua maioria feitas em revistas nacionais, a Scopus apresenta um número maior de publicações realizadas em periódicos internacionais. 


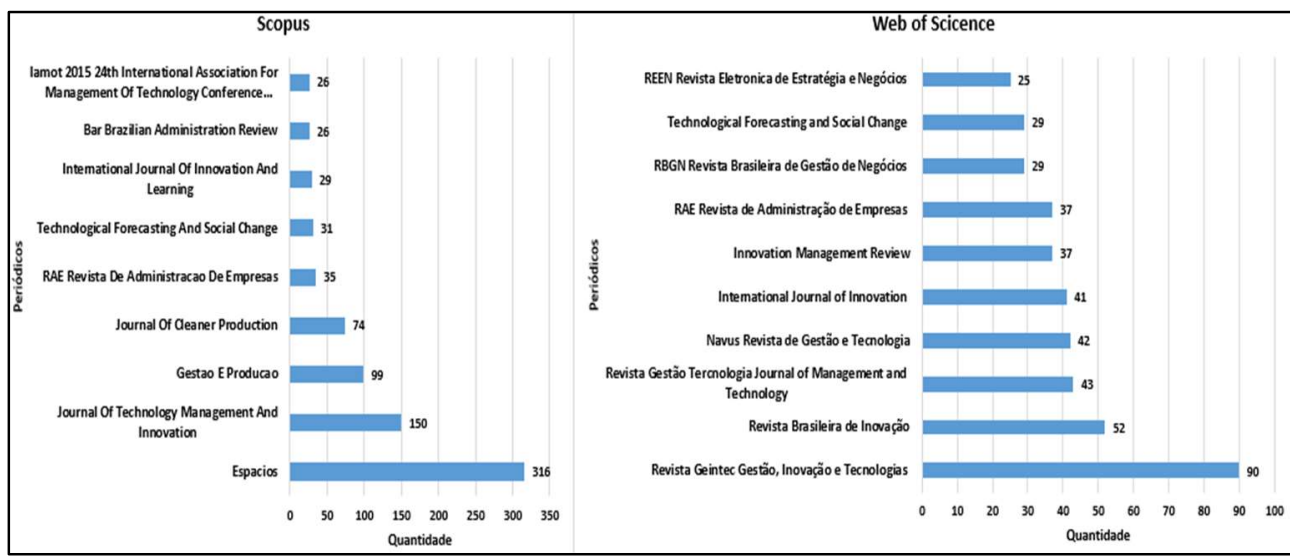

Figura 4: Periódicos indexados com mais publicações científicas brasileiras sobre Inovação (2005 a 2019).

No que diz respeito às universidades que mais publicam nessas bases se destaca a Universidade de São Paulo (USP) ocupando o topo em ambas as plataformas, esta que é considerada a $8^{\circ}$ melhor universidade do mundo pelo ranking do Centro de Estudos em Ciência e Tecnologia (CWTS, na sigla em inglês) a única instituição latino-americana a está entre as 100 melhores do mundo, neste mesmo ranking destacam-se as Universidade Estadual de Campinas (Unicamp), na 183르, bem e a Universidade Federal do Rio Grande do Sul (UFRGS), na 192a posição, universidades que estão entre as 10 que mais publicam sobre a temática da inovação nas bases de dados consultadas. Tais dados são apresentados na Figura 5.



Figura 5: Universidades com mais publicação científica brasileira sobre Inovação (2005 a 2019).

Ainda de acordo com os resultados da Figura 5, Seguindo a USP nos resultados coletados, a Universidade Federal do Rio Grande do Sul (UFRGS), na Scopus, e Universidade de Santa Catarina (UFSC), na Web of Science, destacam fortemente junto à ela as regiões sul e sudeste, e principalmente do Estado de São Paulo, onde há uma concentração sistemática da produção cientifica, pois a cidade de São Paulo concentra $20 \%$ da produção cientifica brasileira (SIDONE et al., 2016), como as regiões que mais produzem conhecimento acerca da temática da inovação no país, quando considerados os dados quantitativos acerca de artigos publicados.

Em relação aos autores com mais publicações, tal como exposto na Figura 6 abaixo, na Web of Science temos Felipe Mendes Borini, professor da Universidade de São Paulo (USP) pesquisador nas áreas de estratégia e inovação global, o fato da USP ser a universidade que mais pública nas bases, ter um pesquisador da instituição nesta categoria corrobora com os dados apresentados anteriormente, aparecendo também em segundo lugar na base Scopus, que tem Eliana Andrea Severo, professora do Programa de Mestrado 
Profissional em Gestão Empresarial (MPGE) do Centro Universitário UniFBV/Wyden, pesquisadora nas áreas de sustentabilidade e inovação, com maior número de publicações, mas que também está presente na Web of Science.

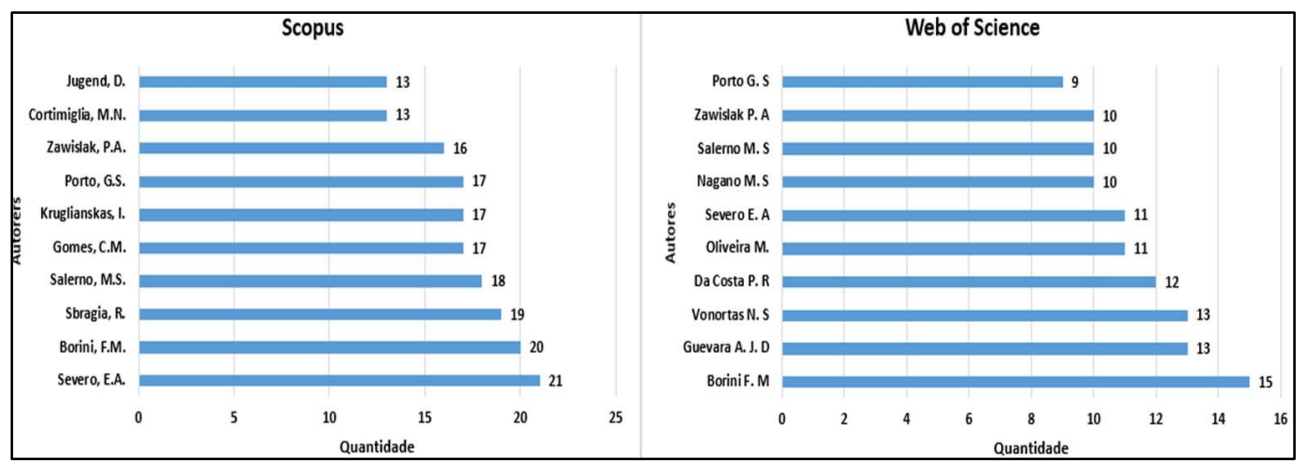

Figura 6: Autores com mais publicação científica brasileira sobre Inovação (2005 a 2019).

No âmbito geral, percebe-se um destaque do estado de São Paulo no que diz respeito às universidades e autores que mais publicam sobre a temática da inovação, o que é reflexo dos investimentos e políticas estaduais voltadas para ciência, tecnologia e inovação, de acordo com o índice FIEC de inovação, São Paulo é o estado mais inovador do Brasil. Além de ser o líder em Investimento Público em Ciência e Tecnologia e Competitividade Global em Setores Tecnológicos (FIEC). Ou seja, os resultados encontrados na pesquisa são fruto de um trabalho desenvolvido no estado de forma consistente no desenvolvimento do seu próprio sistema de inovação (CASSIOLATO et al., 2005).

Ao partir para a análise das publicações mais citadas das bases de dados, olhando primeiramente para as citações dos trabalhos indexados na Scopus, conforme apresentado na Tabela 1, que o artigo mais citado, contendo 718 citações foi produzido no ano de 2013. A partir daí, nota-se um decaimento do número de citações, onde do segundo artigo em diante os textos foram citados menos da metade do primeiro, sendo o segundo citado 212 vezes, e o terceiro somente 177, por exemplo.

Tabela 1: Artigos mais citados na base Scopus

\begin{tabular}{|c|c|c|c|}
\hline TÍTULO & AUTOR & FONTE & CITAÇÕES \\
\hline $\begin{array}{l}\text { Developing a framework for responsible innovation } \\
\text { (2013) }\end{array}$ & $\begin{array}{l}\text { STILGOE, J.; OWEN, R.; } \\
\text { MACNAGHTEN, P. }\end{array}$ & $\begin{array}{l}\text { RESEARCH POLICY Volume: } \\
42 \\
\text { Edição: } 9 \\
\text { Páginas: } 1568-1580\end{array}$ & 718 \\
\hline $\begin{array}{l}\text { Success factors for environmentally sustainable } \\
\text { product innovation: a systematic literature review } \\
\text { (2014) }\end{array}$ & $\begin{array}{l}\text { MEDEIROS, J. F.; RIBEIRO, J. } \\
\text { L. D.; CORTIMIGLIS, M. N. }\end{array}$ & $\begin{array}{l}\text { Journal of Cleaner } \\
\text { Production } \\
\text { Volume: } 65 \\
\text { Páginas: } 76-86\end{array}$ & 212 \\
\hline $\begin{array}{l}\text { Mobile banking: proposing an integrated intention } \\
\text { structure (2010) }\end{array}$ & $\begin{array}{l}\text { PUSCHEL, J.; MAZZON, J. A.; } \\
\text { HERNANDEZ, J. M. C. }\end{array}$ & $\begin{array}{l}\text { Revista Internacional de } \\
\text { Marketing Bancário } \\
\text { Volume: } 28 \\
\text { Edição: } 5 \\
\text { Páginas: } 389-409\end{array}$ & 177 \\
\hline $\begin{array}{l}\text { The central role of human resource management in } \\
\text { the search for sustainable organizations (2008) }\end{array}$ & $\begin{array}{l}\text { JABBOUR, C. J. C.; SANTOS, } \\
\text { F. C. A. }\end{array}$ & $\begin{array}{l}\text { Revista Internacional de } \\
\text { Gestão de Recursos humanos } \\
\text { Volume: } 19 \\
\text { Edição: } 12 \\
\text { Páginas: } 2133-2154\end{array}$ & 168 \\
\hline $\begin{array}{l}\text { Towards "meta-innovation" in Brazil: The evolution } \\
\text { of the incubator and the emergence of a triple helix } \\
\text { (2005) }\end{array}$ & $\begin{array}{l}\text { ETZKOWITZ, H.; MELLO, J. } \\
\text { M. C.; ALMEIDA, M. }\end{array}$ & $\begin{array}{l}\text { Research Policy } \\
34(4), \text { pp. 411-424 }\end{array}$ & 148 \\
\hline
\end{tabular}


O texto mais citado na base de dados, intitulado "Developing a framework for responsible innovation", de autoria de Stilgoe et al. (2013) trata de uma proposição teórica de governança democrática baseada na inovação responsável, onde seriam abordadas soluções para questões sociais e éticas através de um quadro de quadro dimensões: antecipação, reflexividade, inclusão e capacidade de resposta. Assim, percebe-se com esse estudo, a importância que o processo de inovação desempenha para a sociedade, podendo ser não somente um meio para aumentar lucros, como também para melhorar a qualidade de vida e o convívio social da população.

Já com relação aos artigos mais citados que são indexados pela Web of Science, os quais são apresentados na Tabela 2 abaixo, se percebe que o artigo mais citado é o mesmo da Scopus, porém, entre os periódicos indexados pela base, ocorreram somente 624 citações. Tal fato ocorre pela questão das bases indexarem publicações de diferentes periódicos, e no caso em questão, houveram mais citações do artigo em periódicos indexados pela Scopus do que pela Web of Science.

Tabela 2: Artigos mais citados na base Web of Science.

\begin{tabular}{|c|c|c|c|}
\hline TÍTULO & AUTORES & FONTE & CITAÇÕES \\
\hline $\begin{array}{l}\text { Developing a framework for responsible } \\
\text { innovation (2013) }\end{array}$ & $\begin{array}{l}\text { STILGOE, J.; OWEN, R.; } \\
\text { MACNAGHTEN, P. }\end{array}$ & $\begin{array}{l}\text { RESEARCH POLICY Volume: } 42 \text { Edição: } \\
9 \text { Páginas: } 1568-1580\end{array}$ & 624 \\
\hline $\begin{array}{l}\text { Responsible research and innovation: } \\
\text { From science in society to science for } \\
\text { society, with society (2012) }\end{array}$ & $\begin{array}{l}\text { OWEN, R.; MACNAGHTEN, } \\
\text { P.; STILGOE, J. }\end{array}$ & $\begin{array}{l}\text { SCIENCE AND PUBLIC POLICY Volume: } \\
39 \text { Edição: } 6 \text { Páginas: } 751-760\end{array}$ & 452 \\
\hline $\begin{array}{l}\text { Horizontal and vertical relationships in } \\
\text { developing economies: Implications for } \\
\text { SMEs' access to global markets (2008) }\end{array}$ & $\begin{array}{l}\text { MESQUITA, L. } \\
\text { LAZZARINI, S. G. }\end{array}$ & $\begin{array}{l}\text { ACADEMY OF MANAGEMENT JOURNAL } \\
\text { Volume: } 51 \text { Edição: } 2 \text { Páginas: } 359-380\end{array}$ & 171 \\
\hline $\begin{array}{l}\text { The central role of human resource } \\
\text { management in the search for } \\
\text { sustainable organizations (2008) }\end{array}$ & $\begin{array}{l}\text { JABBOUR, C. J. C.; SANTOS, } \\
\text { F. C. A. }\end{array}$ & $\begin{array}{l}\text { INTERNATIONAL JOURNAL OF HUMAN } \\
\text { RESOURCE MANAGEMENT Volume: } 19 \\
\text { Edição: } 12 \text { Páginas: } 2133 \text { - }\end{array}$ & 149 \\
\hline $\begin{array}{l}\text { Towards "meta-innovation" in Brazil: The } \\
\text { evolution of the incubator and the } \\
\text { emergence of a triple helix (2005) }\end{array}$ & $\begin{array}{l}\text { ETZKOWITZ, H.; MELLO, J. } \\
\text { M. C.; ALMEIDA, M. }\end{array}$ & $\begin{array}{l}\text { RESEARCH POLICY Volume: } 34 \text { Edição: } \\
4 \text { Páginas: } 411-424\end{array}$ & 128 \\
\hline
\end{tabular}

Entretanto, diferentemente da base anterior, na Web os Science, o segundo artigo mais citado se aproxima percentualmente no número de citações, tendo até o momento da coleta dos dados o total de 452 citações. $\mathrm{O}$ artigo de autoria dos mesmos autores do primeiro artigo em número de citações em ambas as bases, trata de um tema similar ao anterior, no qual os autores promovem um estudo inicial para entender como a questão da inovação ligada à responsabilidade social está sendo tratada pela política vigente em países da União Europeia (OWEN et al., 2012).

Além disso, é importante salientar que entre os dez artigos mais citados pelas bases, quatro eram comuns em ambas as bases, o que evidencia ainda mais a importância desses artigos para a produção da Inovação, já que apresentam uma abordagem utilizada pela maioria dos estudos indexados por duas das principais bases de dados do mundo.

Ademais, observando a maioria dos artigos citados utilizando-se uma string que se restringe ao Brasil, se percebeu que a maioria das principais referenciais ainda são de autores internacionais, dados os dados coletados e evidenciados nas Tabelas 1 e 2 acima, tendo alguma participação brasileira em alguns desses artigos, porém, predominando a autoria de pesquisadores estrangeiros. Isso evidencia, obviamente, a importância que a temática da inovação tem em países mais desenvolvidos, como na Europa, e que os 
pesquisadores brasileiros ainda recorrem muito a esse material para embasar seus estudos sobre a temática, visto que ainda é uma área, embora promissora e que tenha crescido bastante nos últimos anos, mas que ainda necessidade de apoiar-se em teóricos com maior consolidação para defender suas ideias no meio acadêmico.

\section{CONCLUSÕES}

O presente estudo objetivou compreender o cenário da pesquisa cientifica a respeito da temática inovação, especificamente no contexto após a implementação da Lei da inovação, fato considerado o marco da inovação no país, aparatos políticos tecnológicos, estes, que são fundamentais para o desenvolvimento da ciência, tecnologia e inovação no país.

Não há dúvidas que a lei da inovação impactou diretamente no crescimento da pesquisa cientifica na área de inovação, pois foi criada com intuito de construir um ambiente propício de inovação no país através de parcerias estratégicas, compartilhamento de infraestrutura, capacitação tecnológica, e isso está claro em ambas as bases pesquisadas de 2005 até 2019 é notório o quanto a temática se desenvolveu no país, no entanto apenas essa Lei não é capaz de causar um grande impacto na realidade atual do país, levando em consideração as suas fragilidades como a excessiva rigidez na gestão de recursos humanos, financeiros e materiais.

Porém, quando observamos os estudos mais citados, percebemos que a temática ainda necessita recorrer bastante a fontes e referências internacionais para melhor embasar os estudos realizados aqui no Brasil. Fato que a princípio pode ser considerado deficitário, mas demonstra a preocupação dos pesquisadores brasileiros em construírem a sua própria literatura acerca da inovação a partir de bases teóricas solidas na comunidade internacional, e que poderão trazer grandes frutos para o campo de estudo no âmbito nacional em um futuro próximo.

O papel do governo, portanto, é o subsidiar o desenvolvimento inovativo e tecnológico através da criação de mecanismos que possibilitem o acesso das empresas aos recursos necessários para gerar inovação que são equipamentos, infraestrutura e mão de obra qualificada, e isso só é possível através da criação de aparatos legais, tal como contribuiu a Lei da inovação, mas que também fomente estudos científicos sobre o tema para que o país consiga se tornar autossuficiente na produção tanto do conhecimento inovativo como das próprias ideias geradas à partir dele.

O presente estudo, por se tratar de um estudo bibliométrico e estatístico reconhece as possíveis falhas que possa conter, uma vez que não parte para a análise total de todo o material indexado no mérito de todo o seu conteúdo, embora se apresente como um primeiro norte para buscar compreender como é tratado o tema da inovação no país, principalmente após o advento da Lei da Inovação, utilizada como marco temporal neste estudo.

Como sugestão para estudos futuros, se pode apontar a possiblidade de realizar pesquisas bibliográficas com a produção cientifica sobre inovação das principais universidades que publicam sobre o tema, tal como a Universidade de São Paulo e a Universidade Federal do Rio Grande do Sul, para assim, 
entender mais profundamente acerca da área de concentração e de como o tema inovação é tratado pelos teóricos brasileiros.

\section{REFERÊNCIAS}

AMARAL, E. D.. Tecnologia e Inovação. Montes Claros: IFNMG, 2015.

BUFREM, L. S.; FREITAS, J. L.; SILVEIRA, M.. Políticas de Ciência, Tecnologia e Inovação no Brasil: panorama histórico e contemporâneo. P2P \& INOVAÇÃO, Rio de Janeiro, v.5, n.1, p.6-25, 2018

BRASIL. Lei $\mathbf{n} . \mathbf{1 0 . 9 7 3}$ de $\mathbf{2}$ de dezembro de 2004. Dispõe sobre incentivos à inovação e à pesquisa científica e tecnológica no ambiente produtivo e dá outras providências. Brasília: DOU, 2004.

BRASIL. Lei n. 11.196 de 21 de novembro de 2005. Dispõe sobre incentivos fiscais para a inovação tecnológica. Brasília: DOU, 2005.

CARDOSO, C.. Uma jornada pela inovação. Salvador: EDUFBA, 2010.

CASSIOLATO, J. E.; LASTRES, H. M. M.. Sistemas de inovação e desenvolvimento: as implicações de política. São Paulo em Perspectiva, v.19, n.1, p.34-45, 2005.

CHUEKE, G. V.; AMATUCI, M.. O que é bibliometria? Uma introdução ao Fórum. Revista Eletrônica de Negócios Internacionais. São Paulo, v.10, n.2, p.1-5, 2015.

CNI. Confederação Nacional da Industria. 0 marco legal e os gargalos da Lei n. 13.243 de 2016. Brasília: CNI, 2018.

COSTA, S. E BORINI, F. M.. Inovação Global em Subsidiárias Estrangeiras: o impacto da orientação empreendedora e das redes de empresas. BBR Brazilian Business Review, v.14, n.4, 2017. DOI: https://doi.org/10.15728/bbr.2017.14.4.4

DEPINE, A.; TEIXEIRA, C. S.. Habitats de inovação: conceito e prática. São Paulo: Perse, 2018.
FUCK, M. P.; VILHA, A. P. M.. Inovação tecnológica: da definição à ação. Contemporâneos: Revista de Artes e Humanidades, n.9, p.1-21, 2012.

MOMESSO, A. C.; NORONHA, D. P.. Bibliométrie ou Bibliometrics: o que há por trás de um termo?. Perspectivas em Ciência da Informação, v.22, n.2, p.118-124, 2017.

OCDE. Organização para a Cooperação e Desenvolvimento Econômico. Manual de Oslo: diretrizes para coleta e interpretação de dados sobre inovação. 3 ed. FINEP, 1997.

OWEN, R.; MACNAGHTEN, P.; STILGOE, J.. Responsible research and innovation: from science in society to science for society, with society. Science and Public Policy, Oxford, v.39, n.6, p.751-760, 2012. DOI:

https://doi.org/10.1093/scipol/scs093

SEBRAE. Serviço Brasileiro de Apoio às Micro e Pequenas Empresas. Ecossistemas de empreendedorismo inovadores e inspiradores. Brasília: Sebrae, 2020.

SCHUMPETER, J. A.. Business cycles. New York: McGraw-Hill, 1939.

SIDONE, O. J. G.; HADDAD, E. A.; MENA- CHALCO, J. P.. A ciência nas regiões brasileiras: evolução da produção e das redes de colaboração científica. TransInformação, Campinas, v.28, n.1, p.15-31, 2016.

STILGOE, J.; OWEN, R.; MACNAGHTEN, P.. Developing a framework for responsible innovation. Research Policy, Amsterdã, v.42, n.9, p.1568-1580, 2013. DOI: https://doi.org/10.1016/j.respol.2013.05.008

WELTER, C. V. N.; SAUSE, J. O.; CAPPELLARI, G.. Tipologias de inovação: um estudo em organizações graduadas de incubadoras de base tecnológica. Revista Ibero-Americana de Estratégia, São Paulo, v.18, n.4, p.576-597, 2019.

A CBPC - Companhia Brasileira de Produção Científica (CNPJ: 11.221.422/0001-03) detém os direitos materiais desta publicação. Os direitos referem-se à publicação do trabalho em qualquer parte do mundo, incluindo os direitos às renovações, expansões e disseminações da contribuição, bem como outros direitos subsidiários. Todos os trabalhos publicados eletronicamente poderão posteriormente ser publicados em coletâneas impressas sob coordenação da Sustenere Publishing, da Companhia Brasileira de Produção Científica e seus parceiros autorizados. Os (as) autores (as) preservam os direitos autorais, mas não têm permissão para a publicação da contribuição em outro meio, impresso ou digital, em português ou em tradução. 